\title{
DYNAMIC DIGITAL ASSISTANT USING RASPBERRY PI
}

Komal Nikure

Department of Electronics and Communication, Ballarpur Institute of Technology Ballarpur, India

\begin{abstract}
One of the goals of "'Dynamic Digital Assistant ''is boon for everyone in this new era of $21^{\text {st }}$ century. It has developed for new technology where we can ask question to smart speaker and can interact with IVAs as people do with human. This new technology attracted almost world-wide in many ways like smart phone, laptop computer etc. The purpose of this paper is to interacting between smart speaker and user through the voice command and voice instruction using Raspberry Pi. In this system the two digital assistant operate on a single raspberry pi. Basically python language is used in this system. The main object of this system is to provide the voice information to the user by using the Google assistant or Alexa through the USB speaker or USB microphone in real time mode. In this paper we can operate or control some home automation system by giving voice command to the system. Electric bulb or other electronic device can handle. This paper is based on user input. Location awareness and the ability to access information from a variety of online sources such as weather update, current news, telling days and time, travel assistant, playing songs and events. In this model Raspberry pi $3 B+$ is the principle equipment to execute this model which takes a shot at fundamental contribution of client voice. In this paper speech to text engine (STT) converter is utilized for changing over voice contribution to content code just as Text to speech engine (TTS) is utilized for changing over content to discourse yield.
\end{abstract}

Keywords: Raspberry pi, USB mic, USB speaker, Relay, ESP8266

\section{INTRODUCTION}

We talked about various intelligent personal assistants (IPAs) accessible in the market. These IPAs are enacted by voice directions and can be utilized to control the PDA. In this article, we will attempt to grandstand our potential in our up and coming innovation, brilliant speakers. Basically, keen speakers are versatile speakers that can deliver sound just but on the other hand are shrewd to make home simpler for our everyday tasks. IPA joining with the home speaker empowers them to connect with the general population. Clients can pose inquiries, get recommendations, or by and large converse with this speaker and they can react to genuine answers. A savvy

\author{
Prof. Ashish Manusmare \\ Department of Electronics and Communication, \\ Ballarpur Institute of Technology Ballarpur, India
}

speaker is more than the world has never experienced. The thought may be wonderful or crazy, yet man-made brainpower is making a reality. It is modified to actuate the counterfeit canny voice-enacted individual colleague speaker, switch TV channel, turn on the lights or mood killer other family unit apparatuses change fan speed, read books, take notes, request on the web and retail. Envision every one of these exercises without contacting our advanced mobile phones. These home speakers resemble little robots that live in our home and in the meantime do some work without numerous voice directions. A voice direction framework implies a framework where voice process as info unravels and comprehends the significance of that information it and produce the genuine voice yield. Voice frame work is straightforward for person. In this ongoing year everybody need to their work simple in brief time so for that they alluded to this voice directions framework for satisfy their work or complete their work. Voice order is essentially used to work any gadget to play out any assignment or compose any information or data without utilizing a mouse consoles. Presently, multi day's voice directions are done on a PC with the assistance of ASR (Automatic Speech Recognition) software program. Computerized right hand is produced using virtual individual aide. In this task we use Google collaborator and Amazon alexa and it will be work like remote helper. Google aide is only the brilliant speaker who can converse with individual and carry on like individual nature. It gives the data about the most recent update like date, time, current news, area, current status of any city and all other overall data. Google aide is created by Google in 18 may in the time of 2016.In earlier year Google was introduced on in excess of 400 million gadgets and now it reached out to huge number of electronic gadget including savvy home application vehicles and machine.

Voice order is a direction which can without much of a stretch comprehend to everybody. In this shrewd speaker we can utilize many info voice dialects. Almost 21 dialects are accessible in Google right hand. In this paper we utilize two advanced aide, for example, Google Assistant and Amazon alexa which are interface with a solitary raspberry pi and associate with cloud server. The fundamental work of this shrewd speaker is to expand the correspondence between brilliant speaker and clients financially. It extends their development step by step in programming world like savvy 


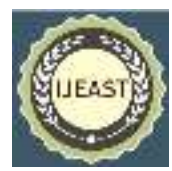

$\mathrm{TV}$, brilliant portable, and some shrewd gadgets. In this venture Amazon alexa is additionally used to communicate with client it likewise gives some ongoing data, for example, news, time, day, current status, and it can set caution. This framework likewise controls to home robotization framework. This innovation is created step by step and it is a lot of gainful for client whose allude this Google collaborator. Google right hand gadget is good with a wide scope of gadget. Along these lines, it can control warming lights and different gadgets with our voice. It can controlled good gadget, for example, Honeywell, whirlphool, Phillips ,Samsung savvy things by utilizing Google colleague additionally it turn light and turn on and off.

\section{PROPOSED WORK}

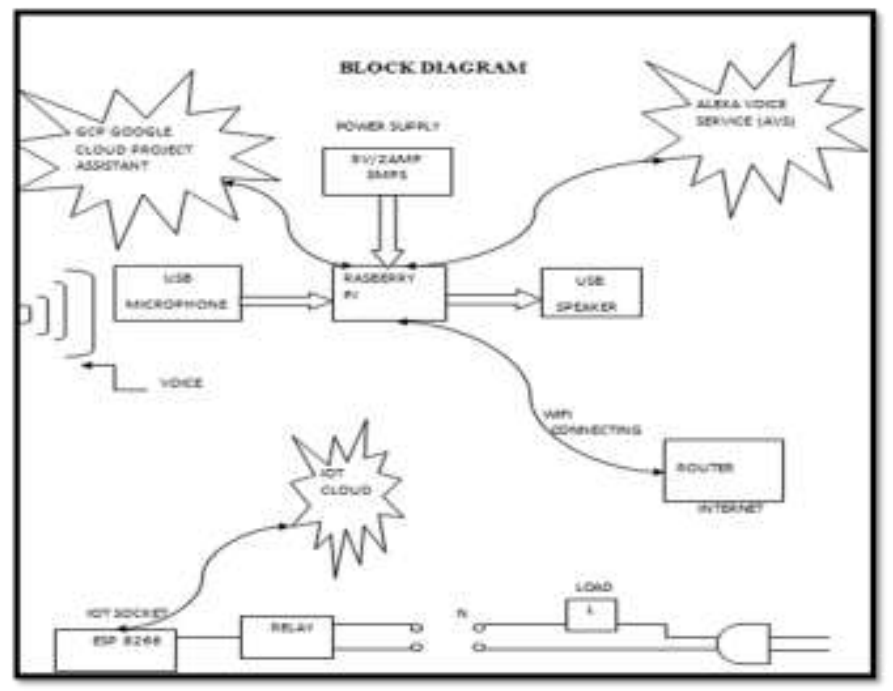

Figure 1: Block Diagram for Proposed System

At first, when the client begins the framework, he utilizes a USB microphone to send the info voice signal. In this framework client talk into the receiver then it will takes info voice from the client and it is nourished to the raspberry pi for handling then the raspberry pi will sustained that information sign to the Google assistant through the raspberry pi. In the wake of getting info voice the Google assistant will contact with cloud server .Cloud server will gather the information from all info gadgets that the framework uses to gather the information from USB mouthpiece at that point contribution from the client it develops inquiries to cloud servers and learning source so as to perform given assignment. After that raspberry pi get that info voice signal from Google cloud server and after that convert it to content then it sends the content to the application in cloud servers to investigate the content and restoring the outcome in USB speaker.

Additionally, Alexa will likewise be functioning as Google assistant. In this framework voice information takes from USB microphone to alexa. In the wake of getting the voice contribution from the microphone alexa contact with cloud server for performing given undertaking and require yield will give in USB speaker. In this framework raspberry pi require $5 \mathrm{v} / 2 \mathrm{amp}$ SMPS. Here raspberry pi is interfacing with the switch through the Wi-Fi or web framework .Router is utilized for sending the information parcels or data between the systems or inside a system or to another system. In this framework some other gadget additionally utilize, for example, ESP8266, IOT Socket, Relay, IOT cloud. The Esp8266 is the controller utilized for controlling this framework. Keen attachment is utilized to associate with other savvy gadget. Hand-off is utilized for on or off the framework. By utilizing this gadget we will work home mechanization framework.

\section{Architecture}

USB Mic USB mic take the voice input data from the user and then it fed to the raspberry pi to performing given task. The raspberry pi does not have an inbuilt microphone. So in this system we use external microphone that connect to raspberry pi.

\section{Raspberry pi}

Raspberry $\mathrm{Pi}$ is a little enough incredible PC to work. Like a normal PC. We are using Raspberry Pi3 model $\mathrm{B}+$ in this project. He has 1 GBRAM; 16GB SD Card is working as storage under, Built-in Wi-Fi and Bluetooth chipsets, HDMI, LAN Ports, $3.5 \mathrm{~mm}$ headphone jacks and 4 USB ports. In this framework raspberry pi interface with PC or PC and afterward it will associate with cloud server for looking through given errand.

\section{Power supply}

In this framework raspberry pi required consistent 5volts $2 \mathrm{~mA}$ power supply. In this framework power supply can either be given through AC supply utilizing a small scale USB charger or through a power bank.

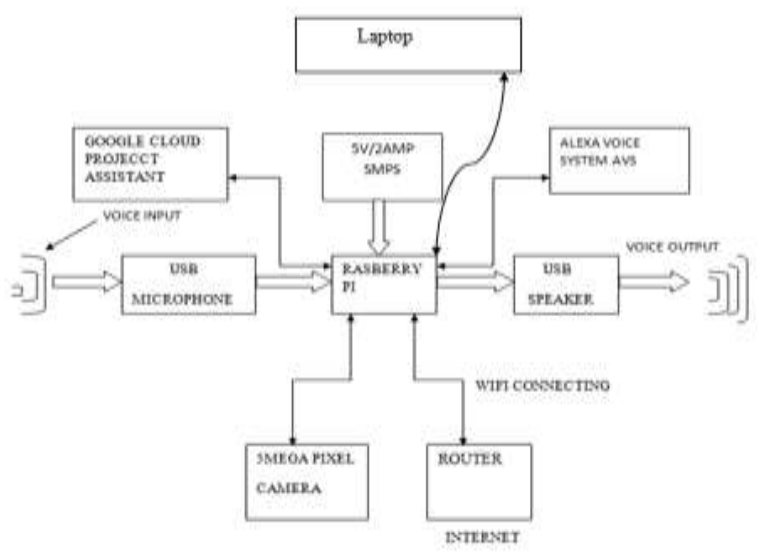


International Journal of Engineering Applied Sciences and Technology, 2019

Vol. 4, Issue 3, ISSN No. 2455-2143, Pages 186-194

Published Online July 2019 in IJEAST (http://www.ijeast.com)

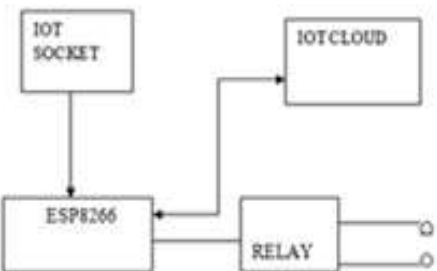

NODE MCU

Figure 2 Architecture of Proposed System

\section{Internet/Wi-Fi/Router}

This system is used to provide internet connection to the voice command system and also the other system. Wi-Fi is utilized to associate the other gadget. By utilizing this web framework the data or any information can advance on Google cloud and alexa cloud for performing given errand.

\section{Speaker}

Speaker is used to provide output voice data to the user. In this framework speaker once the question set forward by the client has been prepared the content of that inquiry is changed over to discourse utilizing the online content to discourse converter. After that sound yield is sent to client by the speaker which is running on sound out.

\section{Relay}

Hand-off is the gadget and it is utilized to ON and OFF the framework. In this framework transfer is fill in as a switch.

\section{Node Mc ESP8266}

ESP8266 is the mix of microcontroller or wifi and it is help to interface the switch through the wifi and controlling the entire framework.

\section{IoT Socket}

Iot attachment is utilized to associate the other gadget to this framework. It is exceptionally valuable for home mechanization framework. This framework can work by utilizing cell phone or other shrewd gadgets.

\section{Laptop}

Workstation or PC is utilized for presentation the info and yield voice direction through the discourse to content transformation by utilizing the VNC watcher. VNC (Vertual Networking Computing) is a graphical work area sharing framework that uses the remote frame buffer remote (RFB) to remotely control another $\mathrm{PC}$.

\section{FLOW CHART}

For the simplicity and for better understanding four flow charts for different system is given below.

\section{Flowchart for Google assistant}

The process and operation of Google assistant system by flowchart is given in figure.

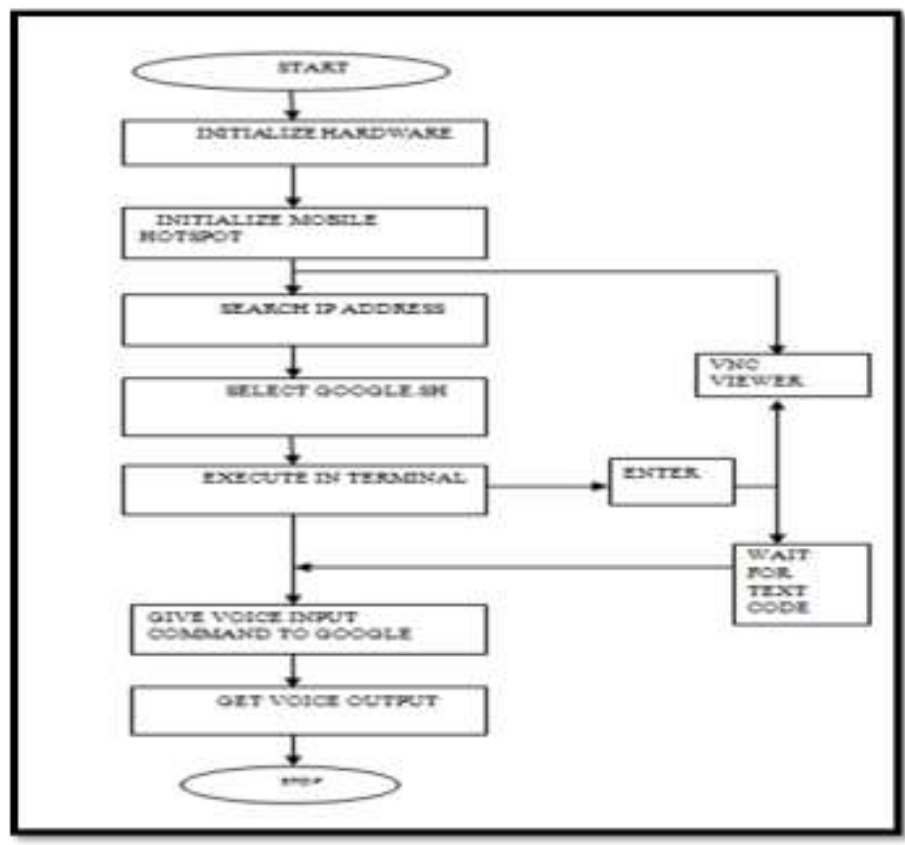

Figure 3: Flowchart for Google assistant

Flowchart for Amazon alexa

The process of operation of Amazon alexa system by flowchart is given in figure.

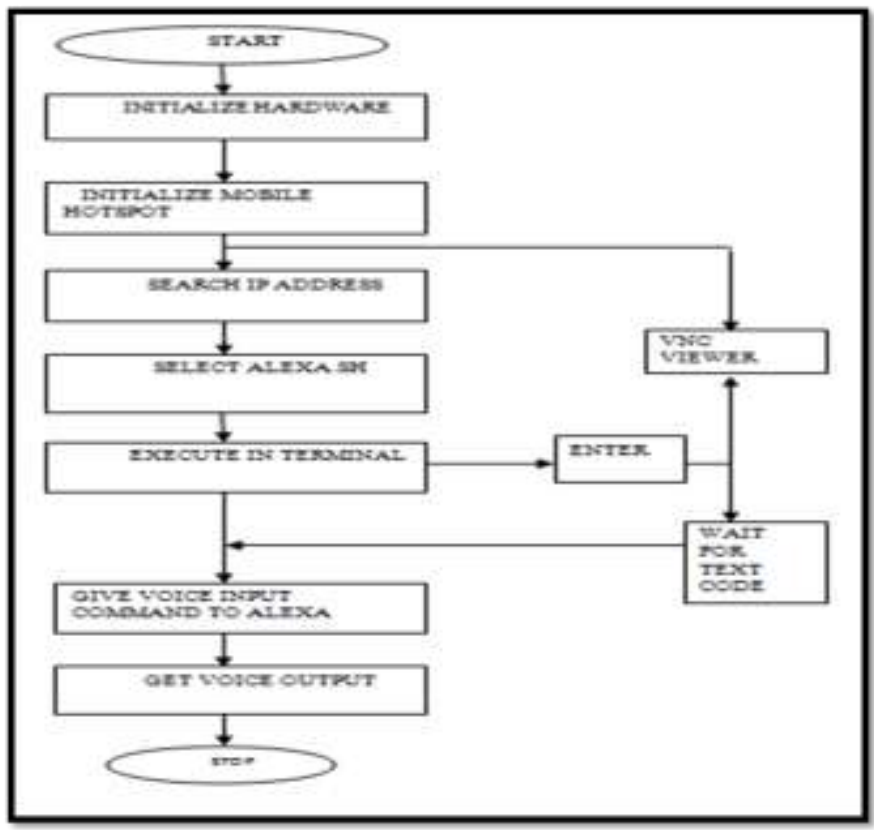

Figure 4: Flowchart for Amazon alexa

Figure 3 and 4 shows the step by step procedure of Google assistant and Amazon alexa system as follows. 
International Journal of Engineering Applied Sciences and Technology, 2019

Vol. 4, Issue 3, ISSN No. 2455-2143, Pages 186-194

Published Online July 2019 in IJEAST (http://www.ijeast.com)

Step 1:

Step 2:

Step 3:

Step 4:

(raspberry pi)

Step 5:

viewer and enter.

Step 6:

enter.

Step 7:

Step 8:

Step 9:

assistant or Amazon alexa from microphone.
Step 10: Get voice output from speaker.

In this advanced right hand framework the working will be same for both Google assistant and just as Amazon alexa from above flowchart. This is the required methodology for working Google assistant and Amazon alexa.

\section{Home automation using BLYNK app}

The process of operation of home automation using BLYNK app by flowchart is given figure. Figure 4.5 shows the step by step procedure of "Home automation using BLYNK app".

Step1: $\quad$ Initialize the hardware.

Step2: $\quad$ Initialize the mobile data.

Step3: $\quad$ Open BLYNK app from mobile.

Step4: Give command to "Turn ON device 1,2,3,4.

This command will send to the Blynk cloud and accept the command and perform given task.

Then the device 1,2,3,4 get "ON".

Similarly, give command for turning "OFF" device 1,2,3,4

Then the device 1,2,3,4 gets "OFF".

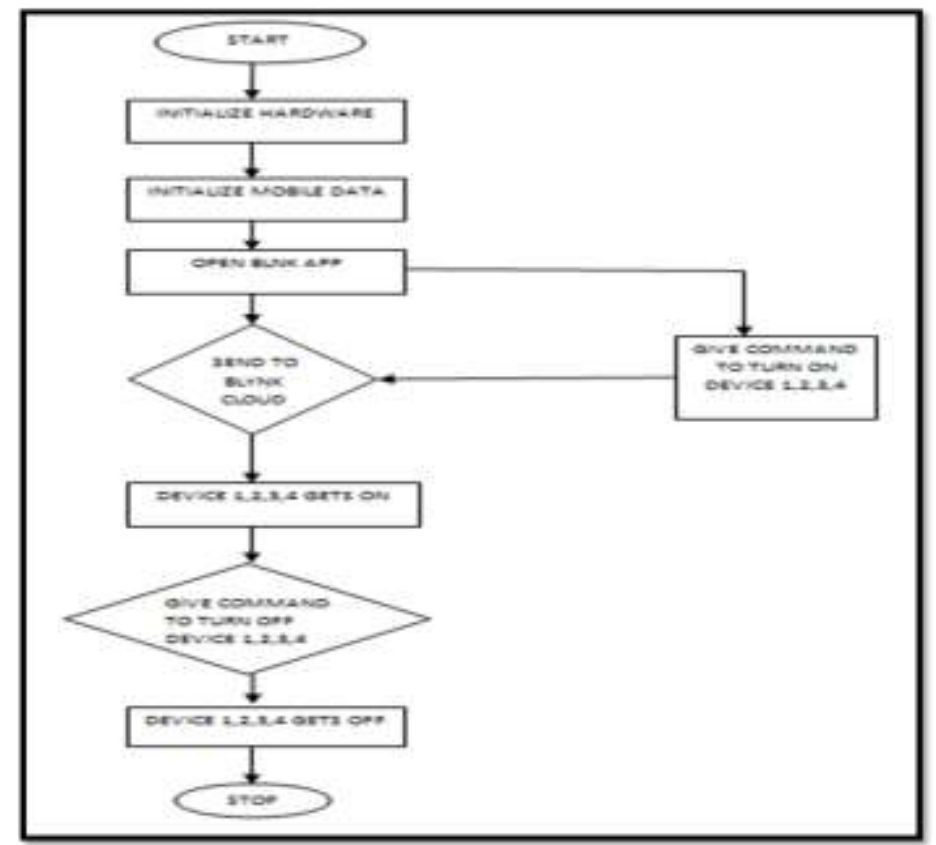

Figure 5: Home automation using BLNYK app

\section{Home automation using Google assistant}

The procedure of activity of home robotization utilizing Google assistant framework flowchart is given in beneath figure. Figure 6 demonstrates the well-ordered methodology of home computerization utilizing Google assistant framework as follows.

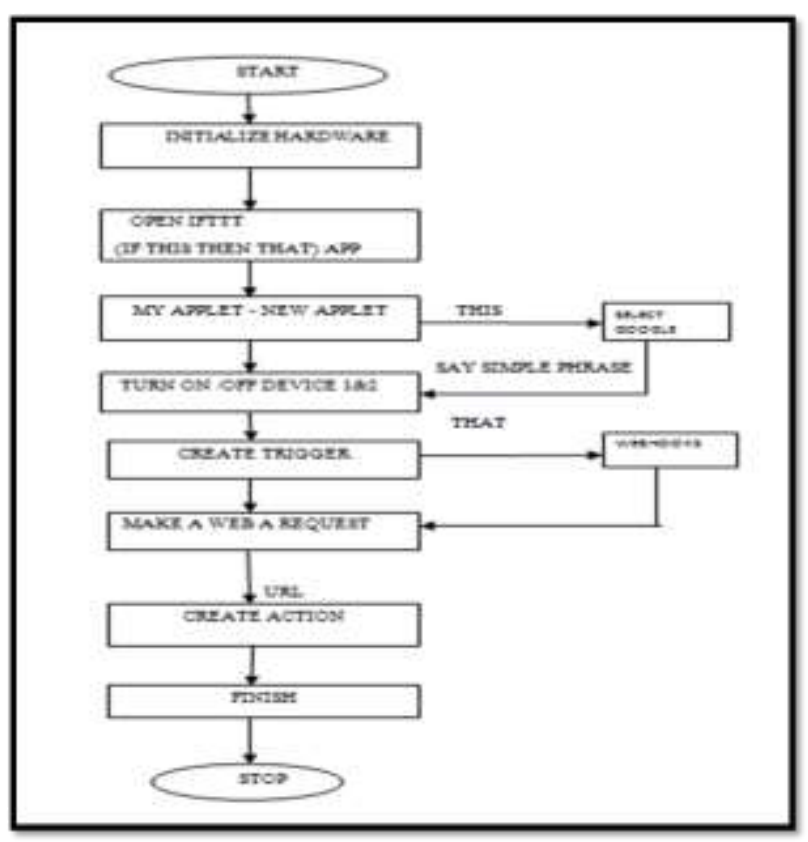

Figure 6: Home automation using Google assistant 
International Journal of Engineering Applied Sciences and Technology, 2019

Vol. 4, Issue 3, ISSN No. 2455-2143, Pages 186-194

Published Online July 2019 in IJEAST (http://www.ijeast.com)

Step 1: $\quad$ Initialize the hardware.

Step 2: $\quad$ Initialize the wifi.

Step 3: $\quad$ Open IFTTT app (If this then that).

Step 4: $\quad$ Select new applet then open my applet.

Step 5: $\quad$ For "This" select "Goggle."

Step 6: $\quad$ Say simple phrase (Turn on /off device 1\&

2).

Step 7: $\quad$ create trigger for Google.

Step 8: $\quad$ For "That" Select webhooks.

Step 9: $\quad$ Make a web request.

copy and paste the IP address and Value $0 / 1$ for ON and OFF for device $1 \& 2$ in URL.

After that create action and then finish.

Give voice command to the Google assistant for turn ON (Ok

Google turn ON device 1 and device 2).

Get the device $1 \& 2$ will ON mode.

Similarly give voice command to Google assistant for turning

OFF (Ok Google turn OFF device 1 and device 2).

Get the device $1 \& 2$ will OFF mode.

\section{Home automation using Amazon alexa}

The process of operation of home automation using Amazon alexa system flowchart is given in below figure. Figure 4.15 shows the step by step procedure of home automation using Google assistant system as follows.

Step 1: $\quad$ Initialize the hardware.

Step 2: $\quad$ Initialize the wifi.

Step 3: $\quad$ Open IFTTT app (If this then that).

Step 4: $\quad$ Select new applet then open my applet.

Step 5: $\quad$ For "This" select "Amazon alexa."

Step 6: $\quad$ Say simple phrase (Turn on /off device $1 \&$

2).

Step 7: $\quad$ create trigger for Alexa.

Step 8: $\quad$ For "That" Select webhooks.

Step 9: $\quad$ Make a web request.

copy and paste the IP address and Value $0 / 1$ for ON and OFF for device $1 \& 2$ in URL.

After that create action and then finish.

Give voice command to the Amazon alexa for turn ON (Alexa trigger turn ON device 3 and device 4 ).

Get the device $3 \& 4$ will ON mode.

Similarly give voice command to alexa for turning OFF (Alexa trigger turn OFF device 3 and device 4).

Get the device $3 \& 4$ will OFF mode.

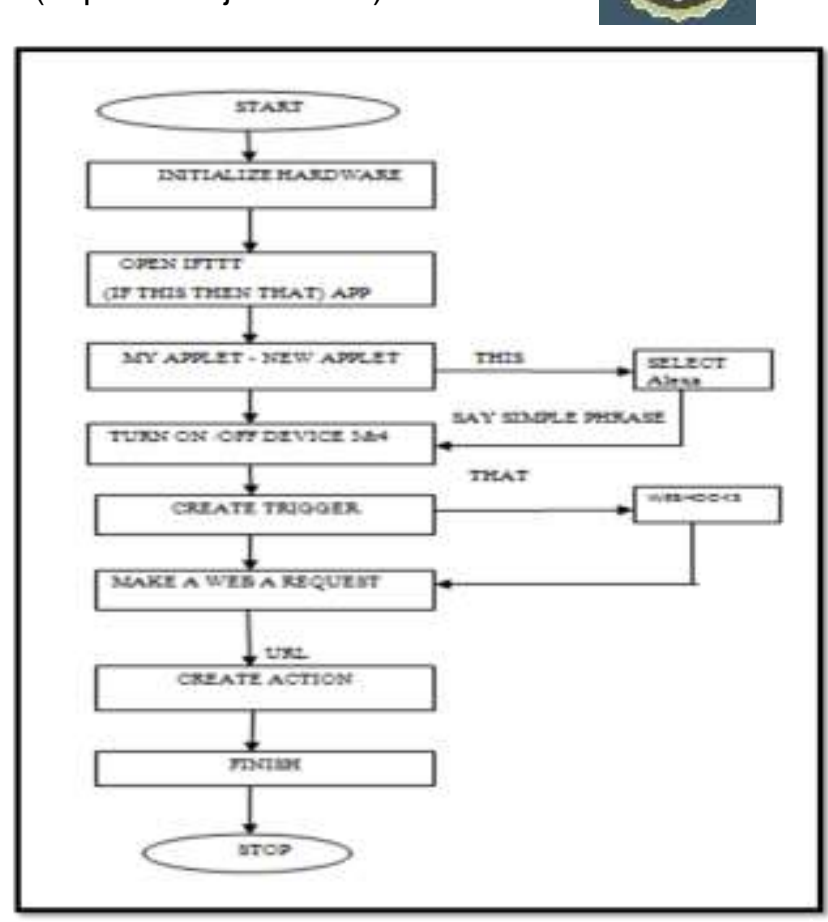

Figure 7: Home automation using Amazon alexa

Hardware Description

The hardware components in this project for three different modules are as follows:

For Digital Assistant

USB Speaker

Raspberry pi $3 \mathrm{~B}+$ module

Mic

USB Cable

Laptop

For Home automation

Raspberry pi

USB cable

Electric bulb

Relay

ESP8266

Speaker/Mic

Laptop

Power supply 5V

For Digital camera

5mega pixel camera

Speaker and Mic

Laptop

USB cable

Raspberry pi

Software used 


\section{International Journal of Engineering Applied Sciences and Technology, 2019 Vol. 4, Issue 3, ISSN No. 2455-2143, Pages 186-194 \\ Published Online July 2019 in IJEAST (http://www.ijeast.com)}

VNC Viewer

Blynk App

IFTTT

Dataplicity

Dialogflow

Arduino IDE

\section{RESULT}

In the figure 8 The VNC watchers is the work area sharing framework programming. It utilized for working Google assistant just as Amazon alexa. In this framework when the client begin to give voice order to the Google assistant through the mic then this voice information direction get from Google collaborator and convert it into content code and search on cloud by utilizing wifi and complete the given errand.

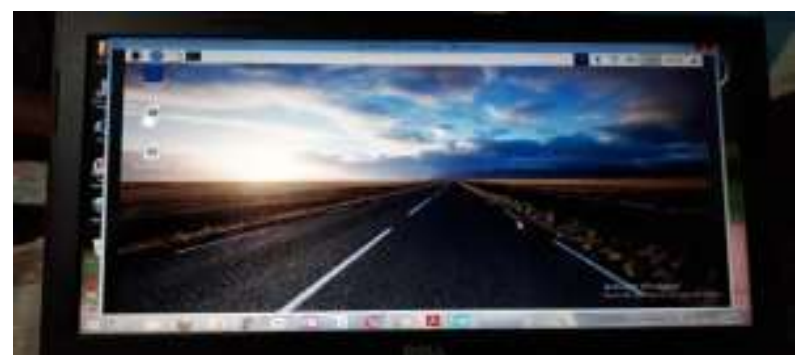

Figure 8: VNC viewer software

The underneath Blynk application is utilized for turning ON and OFF or turning on and off the gadget in home computerization framework. The Blynk application is anything but difficult to working and observing the gadget. By utilizing this application we can work the gadget from other spot. This is the IoT gadget. It can keep running by utilizing other mobile application.

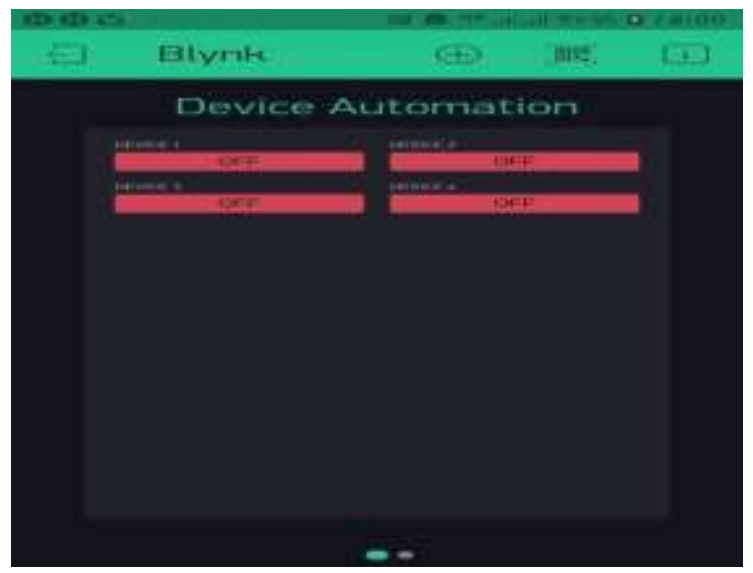

Figure 9: Blynk app in OFF mode

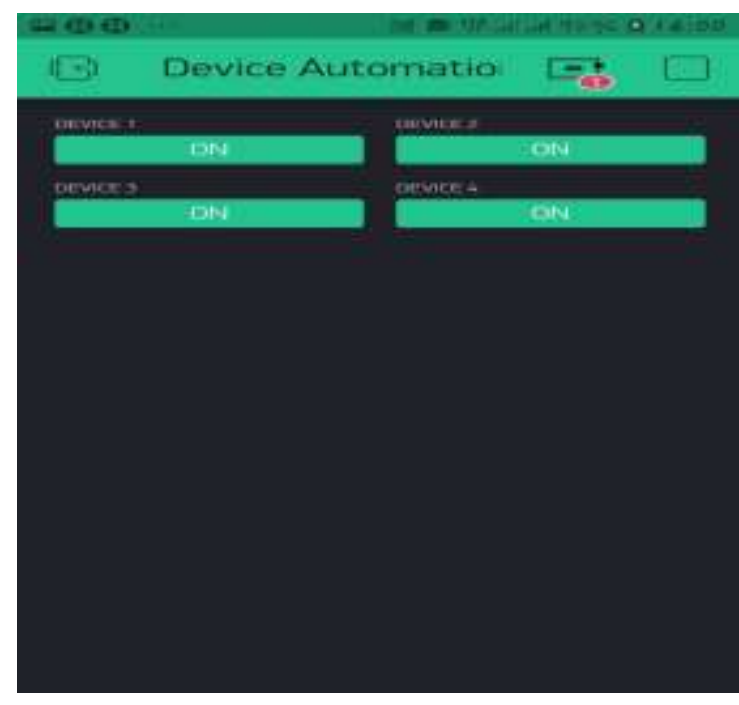

Figure 10: Blynk app in ON mode

- For Google assistant system

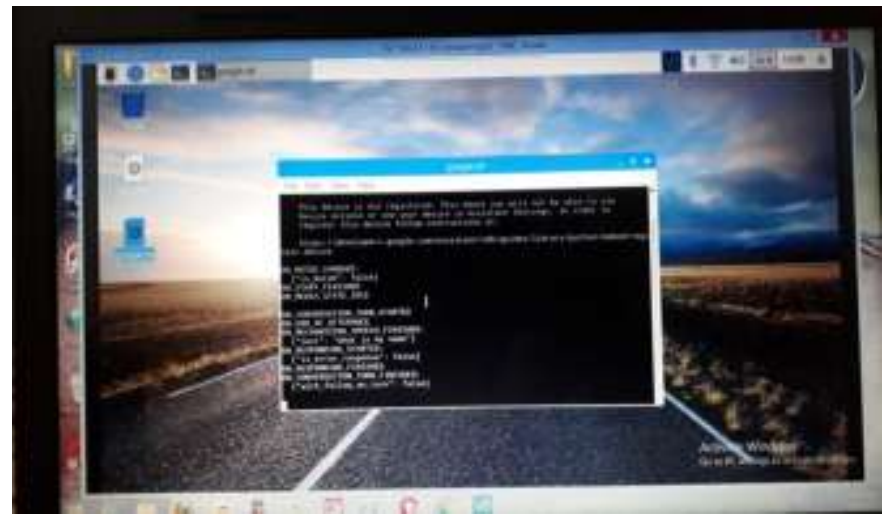

Figure 11: Voice text code for Google assistant

The above outline demonstrates the voice content code for Google assistant. From the above graph the Google assistantis work by utilizing vnc watcher, Raspberry pi $3 \mathrm{~B}+$ module and USB speaker. The VNC watcher ( virtual networking computing) is the graphical work area sharing framework that uses the remote frame buffer protocol (RFB) to remotely control another PC. In this framework Raspberry Pi 3B+ module is interface with the vnc watcher with the assistance of wifi for working Google assistant just as the Amazon Alexa. At first client will utilize the USB mouthpiece for giving voice order to the google assistant it implies that the client will pose the inquiry to the Google. Google will get this voice info direction and convert into content code in the google.sh after that google will look through that content on the google cloud server with the assistance of web. After the pursuit content match on Google cloud the Google right hand will answer back 
International Journal of Engineering Applied Sciences and Technology, 2019

Vol. 4, Issue 3, ISSN No. 2455-2143, Pages 186-194

Published Online July 2019 in IJEAST (http://www.ijeast.com)

to the client its implies that the google give the appropriate response of that question. In this framework answer content code additionally show on the google.sh with the assistance of vnc watcher. The beneath outline demonstrates the voice content code for Amazon alexa. From the above outline the Alexa is work by utilizing VNC watcher, Raspberry pi $3 \mathrm{~B}+$ module and USB speaker. The VNC watcher is the graphical work area sharing framework that uses the RFB to remotely control another PC. In this framework Raspberry Pi 3B+ module is interface with the vnc watcher with the assistance of wifi for working Amazon Alexa.

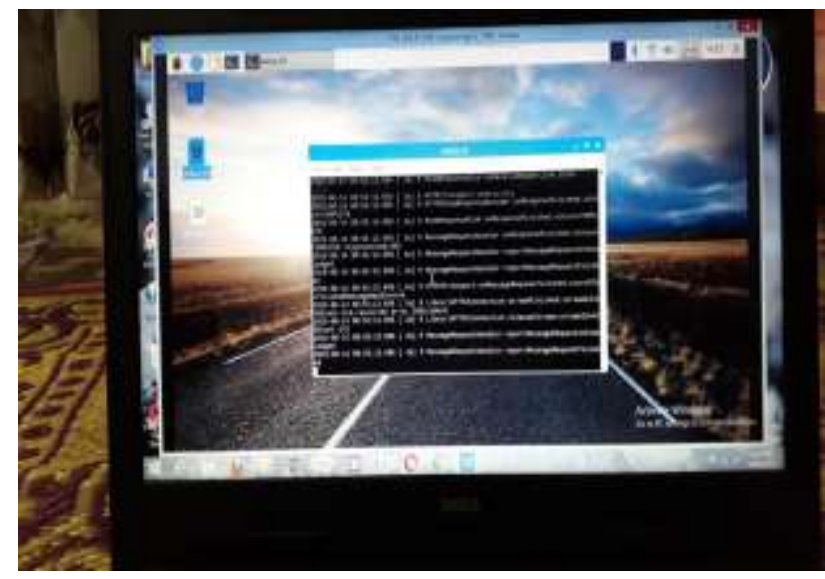

Figure 12: Voice text code for Amazon Alexa

At first client will utilize the USB microphone for giving voice direction to the Amazon alexa it implies that the client will pose the inquiry to the alexa. Alexa will get this voice information order and convert into content code in the google.sh after that alexa will look through that content on the cloud server with the assistance of web. After the inquiry content match on cloud the alexa will answer back to the client its implies that the alexa give the appropriate response of that question. In this framework answer content code additionally show on the alexa.sh with the assistance of vnc watcher.

\section{Home automation using BLYNK app}

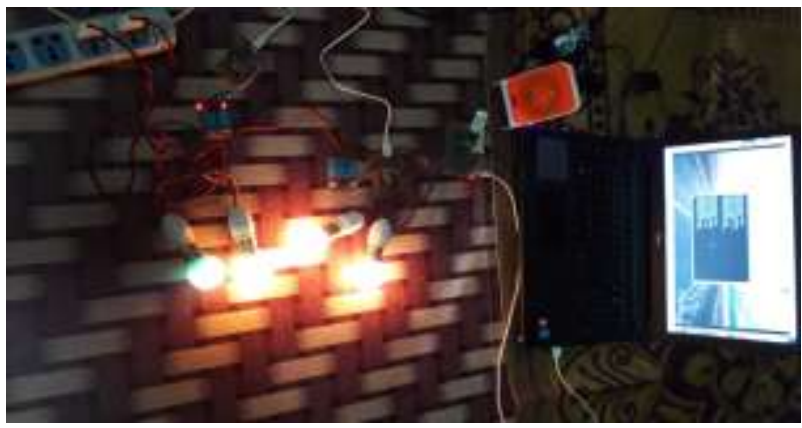

Figure 13: Device 1,2,3,4 ON

Above outline demonstrate the gadget 1,2,3,4 is on by utilizing BLYNK application. In this framework utilized Node MCU ESP8266, Relay and USB power supply for working and observing gadgets by sing the BLYNK application. The hub $\mathrm{MCU}$ is an open source Iot stage it incorporate firmware which keeps running on the ESP8266 wifi soc from ESPress if framework and equipment which depends on the ESP-12 module. In this framework at the yield of ESP8266 the D6 and D7 associate on the transfer R1and R2. On the hand-off board $\mathrm{R} 1$ and $\mathrm{R} 2$ is regularly open and require AC power supply through the switch board.ESP8266 require 5V $2 \mathrm{amp}$ power supply for that we utilized USB power supply. Hand-off R1 and R2 IS NC ordinarily shut is utilized for working gadget it implies that here we are working electric bulb gadget 1and 2 as a working gadget and it comparable for gadget 3and 4 yet it will be work on other esp board and hand-off board it likewise require AC supply for $\mathrm{NO}$ (normally open). This gadget is worked by the blynk is worked by web which is worked with the help of smart phone. After that press the catch for gadget lin the cell phone then this solicitation initially send to the blynk cloud then this solicitation acknowledge through the hotspot or web and again sent back to the blynk application for consequently ON device, comparably for gadget 2, 3 and 4 .

\section{HOME AUTOMATION USING BYLNK APP}

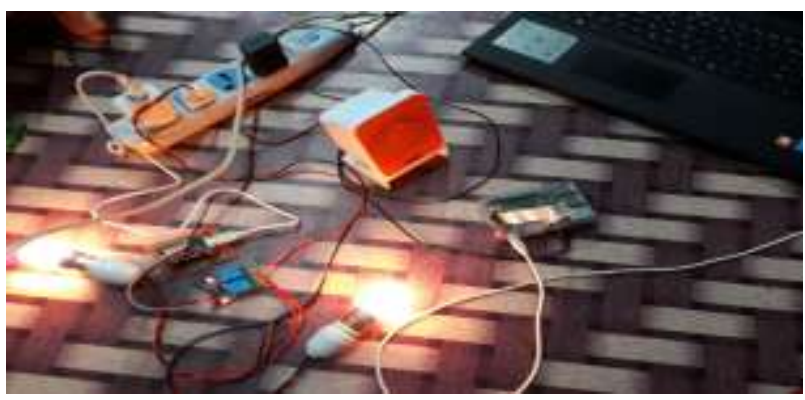

Figure 14: Device $1 \& 2$ ON by voice command

Above outline demonstrate the gadget $1 \& 2$ is on by Google voice order. In this framework we are working home mechanization framework by utilizing voice order to the google assistant. In this framework we utilized IFTTT application for working gadgets. IFTTT is the (IF THIS THEN THAT) framework by utilizing this we can change direction occasion for gadget. At first we open the IFTTT applications at that point select my applet after that select new applet. On the off chance that THIS, THEN THAT first we apply for "THIS" direction and we select google assistant after that in "SAY Simple Phase" we say "TURN ON DEVICE 1". In make trigger we select "THAT" order then we select 


\section{International Journal of Engineering Applied Sciences and Technology, 2019 Vol. 4, Issue 3, ISSN No. 2455-2143, Pages 186-194 \\ Published Online July 2019 in IJEAST (http://www.ijeast.com)}

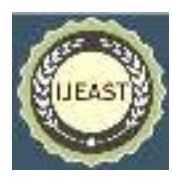

webhooks then we make a web demand after that we reorder the Ip address and volue1for turn ON gadget then we make activity after that wrap up. The comparative procedure is pursuing for gadget 2 for turning $\mathrm{ON}$ and OFF. For turning OFF the gadget we expect esteem "0". After that give voice order to the google assistant "OK GOOGLE TURN ON DEVICE 1" at that point the gadget get turn ON. Thus voice order for turning OFF gadget "OK GOOGLE TURN OFF DEVICE $1 "$ at that point the gadget will get OFF.

\section{Home automation using Amazon Alexa}

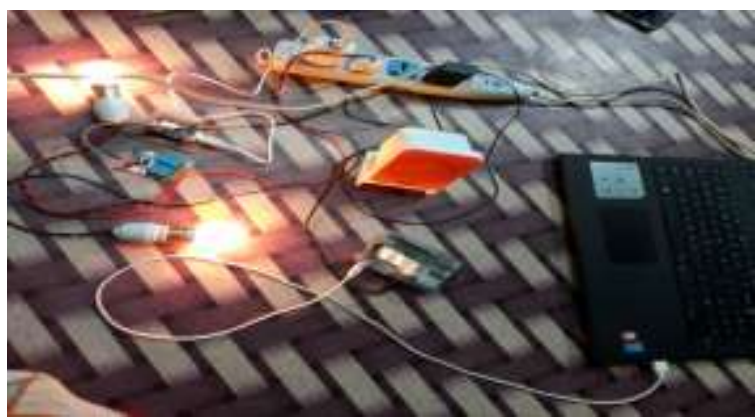

Figure 15: Device $3 \& 4$ ON by voice command

Above chart demonstrate the gadget $3 \& 4$ is on by Alexa voice direction. In this framework we are working home computerization framework by utilizing voice direction to the Amazon alexa. In this framework we utilized IFTTT application for working gadgets. IFTTT is the (IF THIS THEN THAT) framework. By utilizing this we can change order occasion for gadget. At first we open the IFTTT application at that point selects my applet after that select new applet. In the event that THIS, THEN THAT first we apply for "THIS" order and we select Amazon alexa after that in "SAY Simple Phase" we say" TRIGGER TURN ON DEVICE 3". In make trigger we select "THAT" direction then we select webhooks then we make a web demand after that we copy and paste the Ip address and volue1for turn $\mathrm{ON}$ gadget then we make activity after that wrap up.

The comparative procedure is pursuing for gadget 3 for turning ON and OFF. For turning OFF the gadget. We accept esteem "0". After that give voice direction to the Amazon "ALEXA TRIGGER TURN ON DEVICE 3" at that point the gadget get turn ON. Correspondingly voice direction for turning OFF gadget "ALEXA TRIGGER TURN OFF DEVICE 3" at that point the gadget will get OFF.

\section{CONCLUSION}

The proposed framework effectively completes structure and advancement of dynamic computerized colleague utilizing raspberry pi and VNC watcher. It examine effectively on the grounds that all gadgets can be constrained by utilizing brilliant associate and BLYNK application and furthermore this framework utilizes the raspberry pi which is an open source programming that can control numerous gadget automatically. Advanced associate is a quick rising innovation .Now daily's a large portion of the PDA market is driving in android which accompanies Google assistant and Amazon alexa innovation. This strategy is planned in various configurations in future extension. The framework should be secured more and everybody has the option to utilize it from each home or spot.

\section{REFERENCES}

[1] Dhiraj S. Kalyankar. Prof. Dr. P. I Ramteke (2019) "Personal google assistant API assistant system using raspberry pi". e-ISSN:2395-005 p-ISSN:2395-0072 Volume:06,Issue:02 Feb2019,IRJET

[2] Veton Kepuska (2018) "Next generation of virtual personal assistants (Microsoft Cortana, Apple Siri, Amazon Alexa and Google Home)".978-1-5386-46496/18/\$ 31.00 IEEE

[3] Kavya K.S.Math ${ }^{1,}$ Dr. Basavaraj Mathpathi ${ }^{2}$ (2018) "The voice recognition wireless home automaton system based on IoT".(IJTSRO) ISSN NO: 24566470,Volume-2,Issue-5.(Jul.-Aug.)

[4] Kishor kumar R, MS.J.Jayalakshmi, Karthik prasana S (2018) "Python based virtual assistant using raspberry pi". SSRG international jouranal of electronic and communication engineering ISSN: 23488549, Volume5,Issue-7, July

[5] Nidhi Singh (2018) "Intelligent Assistant for Controlling Iot Devices IOSR Journal of Electrical and Electronics Engineering (IOSR-JEEE) e-ISSN: 22781676,p-ISSN: 2320-3331, Volume 13, Issue 4 Ver. I (Jul. - Aug.), PP 30-35

[6] Mallesh S.Jambagi, Basavaraj H, Gagan L, Pratik R Prabhu (2018) "Smart speaker using raspberry pi". ISSN:2456-6470,@IJTSRD Volume-2,Issue-4,(MayJun)

[7] R.Rajyashree ${ }^{1}$, Vineela Kalluru ${ }^{2}$ and Himanshu Kumar ${ }^{3}$ (2018) "Automation using Alexa and Raspberry Pi". eISSN: 2395-0056 ,p-ISSN: 2395-0072,IRJET,Volume05,Issue-10,Oct2018

[8] G. Ashwini, M.Nithish Reddy, R.Paramesh, P.AKHIL (2018) "An intelligent virtual assistant using raspberry pi "ISSN(PRINT)2393-8374(ONLINE):2394-0697 Volume-5.issue-4,

[9] Madhuri Upadhye and R.A. Khan(2017) "Raspberry pi home automation with voice control". ISSN :2454132X,Volume3,Issue6

[10] Chan Zhen Yue and Shum Pig (2017) "Voice activated Smart home design and implementation". $2^{\text {nd }}$ 
International Journal of Engineering Applied Sciences and Technology, 2019

Vol. 4, Issue 3, ISSN No. 2455-2143, Pages 186-194

Published Online July 2019 in IJEAST (http://www.ijeast.com)

international 2017 conference on frontiers of sensor technology 978-1-5090-4860-1/17/\$31.00 IEEE.

[11] Achal S kaundinya,Nikhil S P Atryas, Smrithi Srinivas, Vidhya Keav,Naveen Kumar (2017) "Voice enabled automation using Amazon echo".International Research Journal of Rngineering and technology (IRJET)Volume:04 Issue: 08 August-".e-ISSN:23950056 P-ISSN:2395-0072

[12] Ass. Prof. Emad S. Othman (2017) "Voice Controlled Personal Assistant Using Raspberry Pi" International Journal of Scientific \& Engineering Research Volume 8, Issue 11, November- ISSN 2229-5518

[13] Surinder Kaur $^{1}$,Sanchit Sharma ${ }^{2}$, Utkarsha Jain $^{3}$ and Arpit Raj ${ }^{4}$ (2016) "Voice Command Using Raspberry Pi” An international Journal (ACII) Vol3,No.3,July

[14] Amruta S,Aravind S, Ansu Mathew, Swathy Sugathan,Rajasree R, Priyalakshmi S (2015) "Voice controlled smart home". ISSN:2250-2459,ISO 900/:2008 Certified journal, Vo15 issue 1,January.

[15] Sarthak Jain, Anant Vaibhav, Lovely Goyal, "Raspberry pi based interactive home automation system through E-mail".

[16] Mummaka Sai Srinath ${ }^{1}$, Manepalli Nanda Kishore ${ }^{2}$, M.D. Anto Praveena ${ }^{3}$ (2018) "Interactive home automation system with google assistant". ISSN:13143395, Volume 119 No.12 "Interactive home automation system with google assistant". ISSN:13143395, Volume 119 No.1,14083-14086.

[17] Sonali Sen, Shamik Chakrabarty, Raghav Toshniwal, Ankita Bhaumik d, (2015) "Design of an intelligence voice controlled home automation system," International Journal of Computer Applications (0975 8887), vol. 121 - No.15, July 2015. [18]Foundation, Raspberry Pi, Raspberry Pi 3 Model B specifications. 